\title{
Photothermal Transport of DNA in Entropy-Landscape Plasmonic Waveguides
}

Smith, Cameron; Thilsted, Anil Haraksingh; Pedersen, Jonas Nyvold; Youngman, Tomas Hugh; Dyrnum, Julia C. ; Michaelsen, Nicolai A.; Marie, Rodolphe ; Kristensen, Anders

\section{Published in:}

A C S Nano

Link to article, DOI:

10.1021/acsnano.6b08563

Publication date:

2017

Document Version

Publisher's PDF, also known as Version of record

Link back to DTU Orbit

Citation (APA):

Smith, C., Thilsted, A. H., Pedersen, J. N., Youngman, T. H., Dyrnum, J. C., Michaelsen, N. A., Marie, R., \& Kristensen, A. (2017). Photothermal Transport of DNA in Entropy-Landscape Plasmonic Waveguides. A C S Nano, 4553-4563. https://doi.org/10.1021/acsnano.6b08563

\section{General rights}

Copyright and moral rights for the publications made accessible in the public portal are retained by the authors and/or other copyright owners and it is a condition of accessing publications that users recognise and abide by the legal requirements associated with these rights.

- Users may download and print one copy of any publication from the public portal for the purpose of private study or research.

- You may not further distribute the material or use it for any profit-making activity or commercial gain

- You may freely distribute the URL identifying the publication in the public portal 


\title{
Photothermal Transport of DNA in Entropy- Landscape Plasmonic Waveguides
}

Cameron L. C. Smith, ${ }^{\circledR}$ Anil H. Thilsted, Jonas N. Pedersen, ${ }^{\circledR}$ Tomas H. Youngman, Julia C. Dyrnum, Nicolai A. Michaelsen, Rodolphe Marie, and Anders Kristensen*

Department of Micro- and Nanotechnology, Technical University of Denmark, DK-2800 Kongens Lyngby, Denmark

\author{
Supporting Information
}

ABSTRACT: The ability to handle single, free molecules in lab-on-a-chip systems is key to the development of advanced biotechnologies. Entropic confinement offers passive control of polymers in nanofluidic systems by locally asserting a molecule's number of available conformation states through structured landscapes. Separately, a range of plasmonic configurations have demonstrated active manipulation of nano-objects by harnessing concentrated electric fields. The integration of these two independent techniques promises a range of sophisticated and complementary functions to handle, for example, DNA,
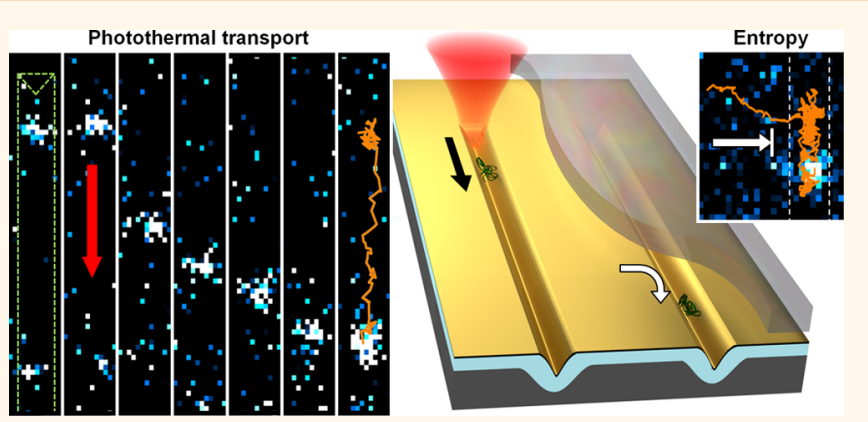
but numerous difficulties, in particular, conflicting requirements of channel size, have prevented progress. Here, we show that metallic V-groove waveguides, embedded in fluidic nanoslits, form entropic potentials that trap and guide DNA molecules over well-defined routes while simultaneously promoting photothermal transport of DNA through the losses of plasmonic modes. The propulsive forces, assisted by in-coupling to propagating channel plasmon polaritons, extend along the $\mathrm{V}$-grooves with a directed motion up to $\approx 0.5 \mu \mathrm{m} \cdot \mathrm{mW}^{-1}$ away from the input beam and $\lambda$-DNA velocities reaching $\approx 0.2$ $\mu \mathrm{m} \cdot \mathrm{s}^{-1} \cdot \mathrm{mW}^{-1}$. The entropic trapping enables the V-grooves to be flexibly loaded and unloaded with DNA by variation of transverse fluid flow, a process that is selective to biopolymers versus fixed-shape objects and also allows the technique to address the challenges of nanoscale interaction volumes. Our self-aligning, light-driven actuator provides a convenient platform to filter, route, and manipulate individual molecules and may be realized wholly by wafer-scale fabrication suitable for parallelized investigation.

KEYWORDS: channel plasmon polariton, photothermal effect, entropic trapping, V-groove, nanofluidics, nanoconfined DNA

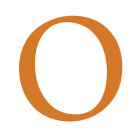

ver the last two decades, nanofluidics ${ }^{1-4}$ has cemented its role as an enabling platform for stateof-the-art DNA analysis tools ${ }^{5,6}$ as it can isolate individual molecules in mass-fabricated lab-on-a-chip devices while supporting many practical solutions for manipulation and detection. Central to nanofluidics is nanoconfinement, in which a polymer restricted below its radius of gyration acts to maximize its entropy, leading to a variety of diverse molecular dynamics. ${ }^{3}$ Several important examples of these can be realized by variation of the structural cross section, such as molecule trapping sites, ${ }^{7,8}$ directed molecular self-organization, ${ }^{9}$ or influenced translocation behavior. ${ }^{10}$ Moreover, channel dimensions close to DNA's persistence length $(\sim 50 \mathrm{~nm})$ cause molecular elongation, allowing fluorescent imaging techniques to capture DNA sequence information ${ }^{5,6,11}$ or study its physical aspects. ${ }^{1,12}$ Considering these features, nanoconfinement represents a compelling technique to handle individual DNA molecules, although its passive nature lacks important capabilities on its own, such as controlled delivery of single molecules, for advanced DNA processing.
In parallel, plasmon-enhanced nanomanipulation is a significant research area that seeks to harness the ability of plasmons to support highly focused electric fields for efficient transfer of momentum to nano-objects. ${ }^{13-16}$ Plasmonics is attractive for lab-on-a-chip applications because it offers localization down to the single-particle level and can be actively controlled by off-chip light sources. ${ }^{17}$ In particular, plasmonic photothermal techniques have made noteworthy progress by exploiting the heat from Ohmic losses to generate controlled fluid motion for highly effective trapping and sorting operations. ${ }^{18-23}$ However, no precedent exists that combines plasmonic transport schemes with entropic confinement capabilities due to the competing requirements of channel height. On one hand, entropic trapping demands sizes smaller than the radius of gyration $R_{\mathrm{g}}$ of the molecule (for $\lambda$-DNA, $R_{\mathrm{g}}=$ $0.56 \mu \mathrm{m}$; see section 1 of the Supporting Information). On the

Received: December 21, 2016

Accepted: April 25, 2017

Published: April 28, 2017 


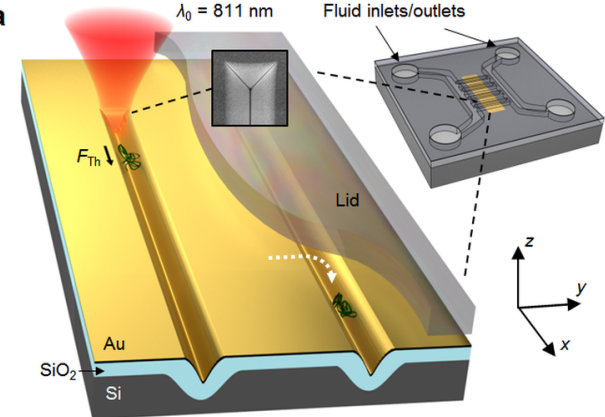

b

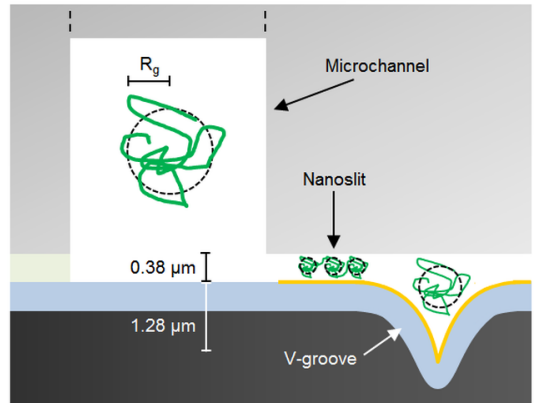

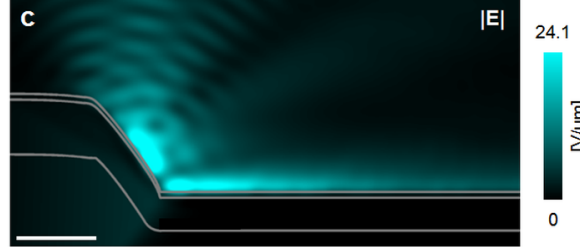
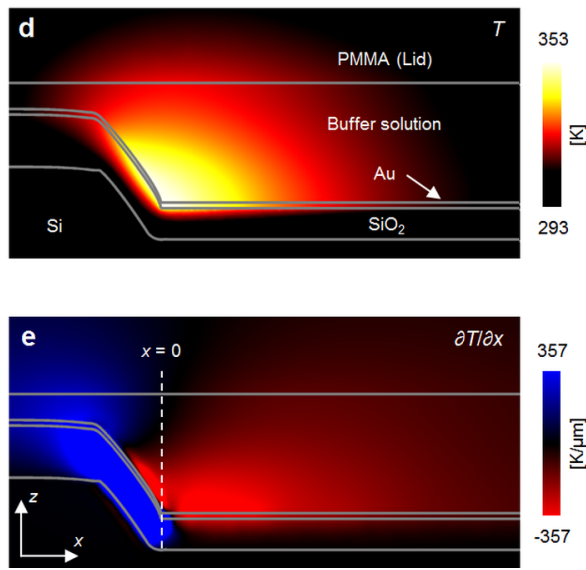

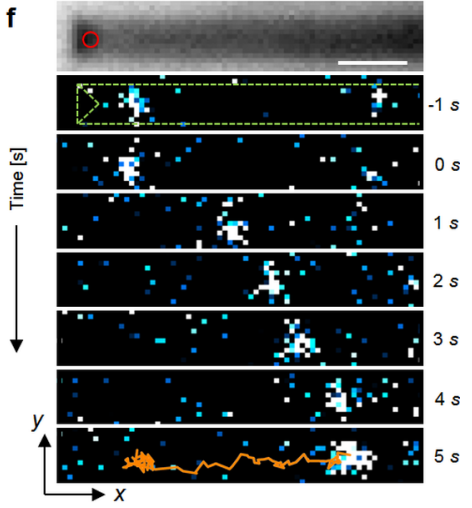

g

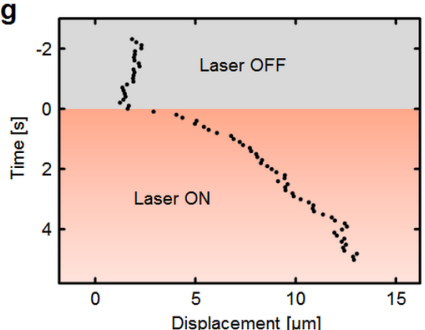

Figure 1. (a) Device and concept illustration: Normally incident light focused onto the V-groove nanomirrors excites plasmons, which generate heat along the grooves. Particles within the temperature gradients experience photothermal transport, in which DNA molecules will exclusively route along the entropic potential of the V-groove structures (see Supporting Information video 1 ). (b) Depiction of the entropy of a $\lambda$-DNA molecule at different locations in the fluidic system. The molecule is unconfined in the microchannel, slightly confined inside the V-groove, and tightly confined in the nanoslit where it becomes a series of connected blobs. Not to scale. (c) Calculation of the normalized electric field along the $x z$-plane showing the excitation of a CPP mode in the V-groove via normal illumination of the termination mirror (1.1 $\mu \mathrm{m}$ spot size, $\lambda_{0}=811 \mathrm{~nm}$, laser power $30 \mathrm{~mW}$ ). The scale bar is $1 \mu \mathrm{m}$. (d) Steady-state temperature distribution corresponding to (c). (e) Partial gradient of the temperature in $(\mathrm{d}), \partial T / \partial x$, representing the component responsible for the thermophoretic force along the groove axis. (f) Top panel: bright-field image of a nanofluidics-integrated plasmonic V-groove. The red circle indicates the position of the incident laser spot. The scale bar is $4 \mu \mathrm{m}$. Lower panels: time series of fluorescent images showing the motion of a single $\lambda$-DNA molecule in a plasmonic $\mathrm{V}$ groove (dashed green line) for the laser parameters of $(\mathrm{c}-\mathrm{e})$ applied from $t=0$. The orange line is the tracked path over $-5 \mathrm{~s} \leq t \leq 5 \mathrm{~s}(\Delta t=$ $0.1 \mathrm{~s})$. (g) Displacement along the groove axis of the DNA molecule presented in (f) versus time.

other hand, plasmonic transport schemes to date have required channel heights larger than one micron in order to avoid too large hydrodynamic resistances that would otherwise stymie carrying fluid flows. For these reasons, precise and highthroughput biopolymer handling has remained out of reach.

In this article, we show that plasmonic V-grooves, integrated within a nanofluidic system, offer a particular and capable method of achieving directed DNA transport along entropic routes via an efficient, plasmon-induced photothermal effect that does not rely primarily on fluid flow. The V-groove waveguides, ${ }^{24,25}$ typically considered in research for nanophotonic circuitry ${ }^{2-28}$ or quantum plasmonics, ${ }^{29,30}$ here serve two alternative and distinct purposes: (1) defining entropic potentials for biopolymers to self-align and guide along and (2) supporting plasmonic modes whose losses generate a temperature gradient ${ }^{31}$ sufficient for controlled photothermal transport. $^{32-37}$ Furthermore, the configuration offers competitive specifications compared to existing nanomanipulation schemes as it can avoid direct photodamage of biomolecules, ${ }^{19}$ does not lead to aggregation, ${ }^{13,38}$ provides propulsion over long distances, ${ }^{14,19,20}$ and, due to the selective entropic filtering, can expedite the loading mechanism by fluid flow rather than relying on diffusion. ${ }^{19,20}$

\section{RESULTS AND DISCUSSION}

Working Principle. The operation of our device is illustrated in Figure 1a, in which a normally incident laser beam focused on a V-groove nanomirror deterministically transports biopolymers in the device plane along the entropic potential of the waveguide. To elucidate the role of entropy, which is governed by the local channel size, the availability of possible molecular conformation states is depicted for different regions of the fluidic system in Figure $1 \mathrm{~b}$. The free energy (inverse entropy) of a $\lambda$-DNA molecule is lowest when it is unconfined in the inlet/outlet microchannels and may assume its full radius of gyration, $R_{g} \approx 0.5 \mu \mathrm{m}$. Inside the nanoslit, the molecule becomes a series of connected blobs ${ }^{39}$ with a reduced availability of conformation states and high free energy, $E_{\mathrm{NS}}$, given by

$$
E_{\mathrm{NS}} / k_{\mathrm{B}} T=A_{\mathrm{NS}}(h, P) L_{\mathrm{p}}
$$

where $h$ is the nanoslit height, $P$ is the polymer persistence length, $A_{\mathrm{NS}}$ is a geometric factor ${ }^{9}$ of the nanoslit given by $A_{\mathrm{NS}} \sim$ $P / h^{2}$, and $L_{\mathrm{p}}$ is the polymer contour length. For $\lambda$-DNA with a $1: 10$ staining ratio of YOYO- 1 dye used in our experiments, we estimate $P \approx 57 \mathrm{~nm}$ and $L_{\mathrm{p}} \approx 19 \mu \mathrm{m}$ based on reported values in literature and evidence that both $P$ and $L_{\mathrm{p}}$ are increased by the same factor according to their staining ratio, ${ }^{9,40}$ resulting in $E_{\mathrm{NS}} \approx 7.5 k_{\mathrm{B}} \mathrm{T}$ for $h=380 \mathrm{~nm}$. Inside an embedded V-groove, the molecule's free energy, $E_{\mathrm{VG}}$, is estimated based on previous work ${ }^{9}$ by

$$
E_{\mathrm{VG}} / k_{\mathrm{B}} T=A_{\mathrm{VG}}\left(h, V_{\mathrm{d}}, V_{\mathrm{w}}, V_{\mathrm{l}}, P\right) L_{\mathrm{p}}
$$

with the corresponding geometric factor defined as 

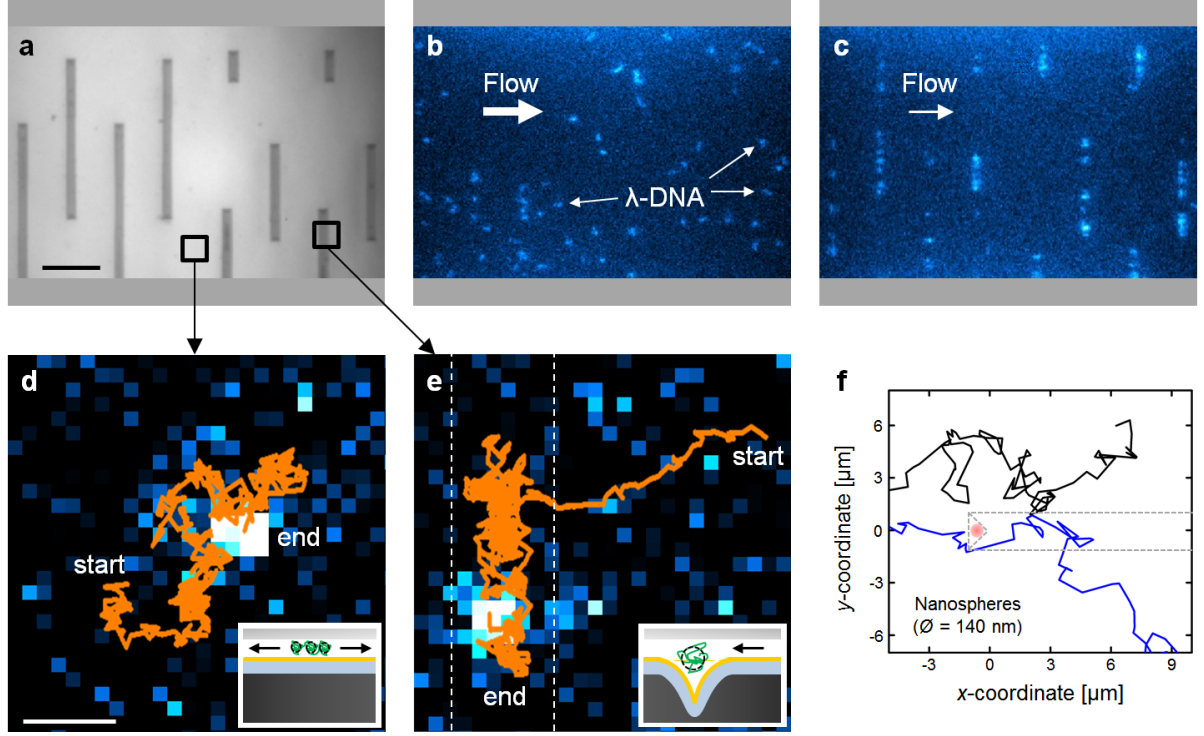

Figure 2. (a) Bright-field image of the nanofluidics-integrated V-grooves. The upper and lower gray sections indicate the sidewalls of the nanoslit. The scale bar is $20 \mu \mathrm{m}$. (b,c) Fluorescent images of the same region as in (a) showing the distribution of $\lambda$-DNA molecules (bright spots; white arrows indicate three examples). Pressure-driven flow delivers the DNA throughout the nanofluidics system: in (b), the flow $(v=$ $\left.1.9 \mu \mathrm{m} \cdot \mathrm{s}^{-1}\right)$ causes the DNA to escape the entropic traps of the V-grooves, whereas in (c), the flow is sufficiently slow $\left(v=0.3 \mu \mathrm{m} \cdot \mathrm{s}^{-1}\right)$ to enable controlled loading of the V-grooves (see Supporting Information video 2). (d,e) Tracked particle traces of $\lambda$-DNA molecules without pressure-driven flow over a duration of $50 \mathrm{~s}$ at the nanoslit locations specified in (a). (d) For the flat surface region $(0.38 \mu \mathrm{m}$ slit height), the DNA molecule diffuses randomly in two dimensions. The scale bar is $2 \mu \mathrm{m}$. (e) Adjacent to a V-groove, a DNA molecule may randomly enter the V-groove trap and subsequently remain. Once inside the V-groove, the molecule diffuses exclusively along the groove axis. (f) Two fixedshape nanospheres of $140 \mathrm{~nm}$ diameter enter the vicinity of a V-groove (dashed gray lines) via Brownian motion, but they are not entropically trapped by the structure. Additionally, the thermal gradient generated by an incident laser beam of $61 \mathrm{~mW}$ has less influence on the trajectories of nanospheres along the V-groove than on the trajectories of $\lambda$-DNA due to the differences in Soret coefficients.

$$
A_{\mathrm{VG}} \sim P\left[\frac{1}{\left(h+V_{\mathrm{d}}\right)^{2}}+\frac{1}{V_{\mathrm{w}}^{2}}+\frac{1}{V_{\mathrm{l}}^{2}}\right]
$$

where $V_{\mathrm{d}}$ is the effective depth of the V-groove, $V_{\mathrm{w}}$ is the wedge-to-wedge width of the $\mathrm{V}$-groove, and $V_{1}$ is the length of the V-groove. Considering $V_{\mathrm{d}}=0.65 \mu \mathrm{m}$ (root-mean-square depth), $V_{\mathrm{w}}=1.95 \mu \mathrm{m}$ (SEM images), and $V_{1}$ set as infinite due to $V_{1} \gg R_{\mathrm{g}}, E_{\mathrm{VG}}=1.3 k_{\mathrm{B}} \mathrm{T}$. The lateral trapping potential of the embedded V-groove for $\lambda$-DNA can be expressed as the difference in free energy between the $\mathrm{V}$-groove and the nanoslit: $\Delta E=E_{\mathrm{NS}}-E_{\mathrm{VG}}=6.2 k_{\mathrm{B}} \mathrm{T}$. This value remains constant along the waveguide axis.

The mechanism of photothermal transport via incident light to the propulsion of DNA is conveyed in the series of $3 \mathrm{D}$ finite element method (FEM) calculations in Figure 1c-e. Figure 1c shows the time-averaged, normalized electric field ( $\mid \mathrm{El})$ viewed along the plane bisecting the waveguide, in which normal illumination of a focused beam $(1.1 \mu \mathrm{m}$ spotsize, free-space wavelength $\lambda_{0}=811 \mathrm{~nm}$, elliptical polarization corresponding to experiments) on the nanomirror launches plasmonic modes in the liquid-cladding gold $\mathrm{V}$-groove. The subsequent heating of the system from the dissipation of plasmons is represented in Figure $1 \mathrm{~d}$, which, assisted by propagating channel plasmon polaritons (CPPs), extends along the waveguide. The total coupling efficiency of light to heat-generating plasmons is calculated to be $\eta=20.4 \%$, with material-based absorption contributing an additional $4.1 \%$. The associated temperature gradient, $\partial T / \partial x$, of Figure $1 \mathrm{e}$ is responsible for the thermophoretic force.

A representative experimental trajectory of entropy-guided photothermal propulsion of DNA is shown in Figure 1f,g. The top panel of Figure 1f is a bright-field image of the nanofluidicsintegrated V-groove, with the red circle indicating the position of the incident beam. Beneath, a time series of fluorescent images shows the motion of a single $\lambda$-DNA molecule guided along a $\mathrm{V}$-groove (dashed green line) for the laser parameters given in Figure 1c-e applied from time $t=0$. The orange line is the tracked path over $-5 \mathrm{~s} \leq t \leq 5 \mathrm{~s}$ (time lapse $\Delta t=0.1 \mathrm{~s}$ ), which remains confined to the $\mathrm{V}$-groove for the duration. Figure $1 \mathrm{~g}$ shows the displacement of the $\lambda$-DNA represented in Figure if along the groove axis versus time. The molecule undergoes Brownian motion in the $\mathrm{V}$-groove before the incident laser excites plasmons that initiate directed transport and propel the $\lambda$-DNA along the waveguide.

Entropic Confinement. An important feature of the entropic landscape is the on-demand loading and unloading of flexible molecules enabled by the open-channel structure of the embedded V-grooves. Figure $2 \mathrm{a}-\mathrm{c}$ highlights this capability, for which controlled transverse fluid flow performs the operation. Figure 2a shows a bright-field image of the nanofluidics-integrated V-grooves under study. Figure $2 b, c$ shows fluorescent images of the same region as in Figure 2a, displaying the distribution of $\lambda$-DNA molecules (bright spots). Pressure-driven flow delivers the DNA throughout the nanofluidics system: in Figure $2 \mathrm{~b}$, the flow (velocity $v=1.9$ $\mu \mathrm{m} \cdot \mathrm{s}^{-1}$ ) carries the DNA over the entropic trap of the embedded V-grooves, whereas in Figure $2 c$, the flow is sufficiently slow $\left(v=0.3 \mu \mathrm{m} \cdot \mathrm{s}^{-1}\right)$ to allow loading of DNA in the V-grooves.

Figure 2 d,e presents the diffusion behavior of $\lambda$-DNA in the integrated nanofluidic system without pressure-driven flow over a duration of $50 \mathrm{~s}$ (start-end) at the nanoslit locations specified in Figure 2a. In Figure 2d, the $\lambda$-DNA diffuses randomly in two 

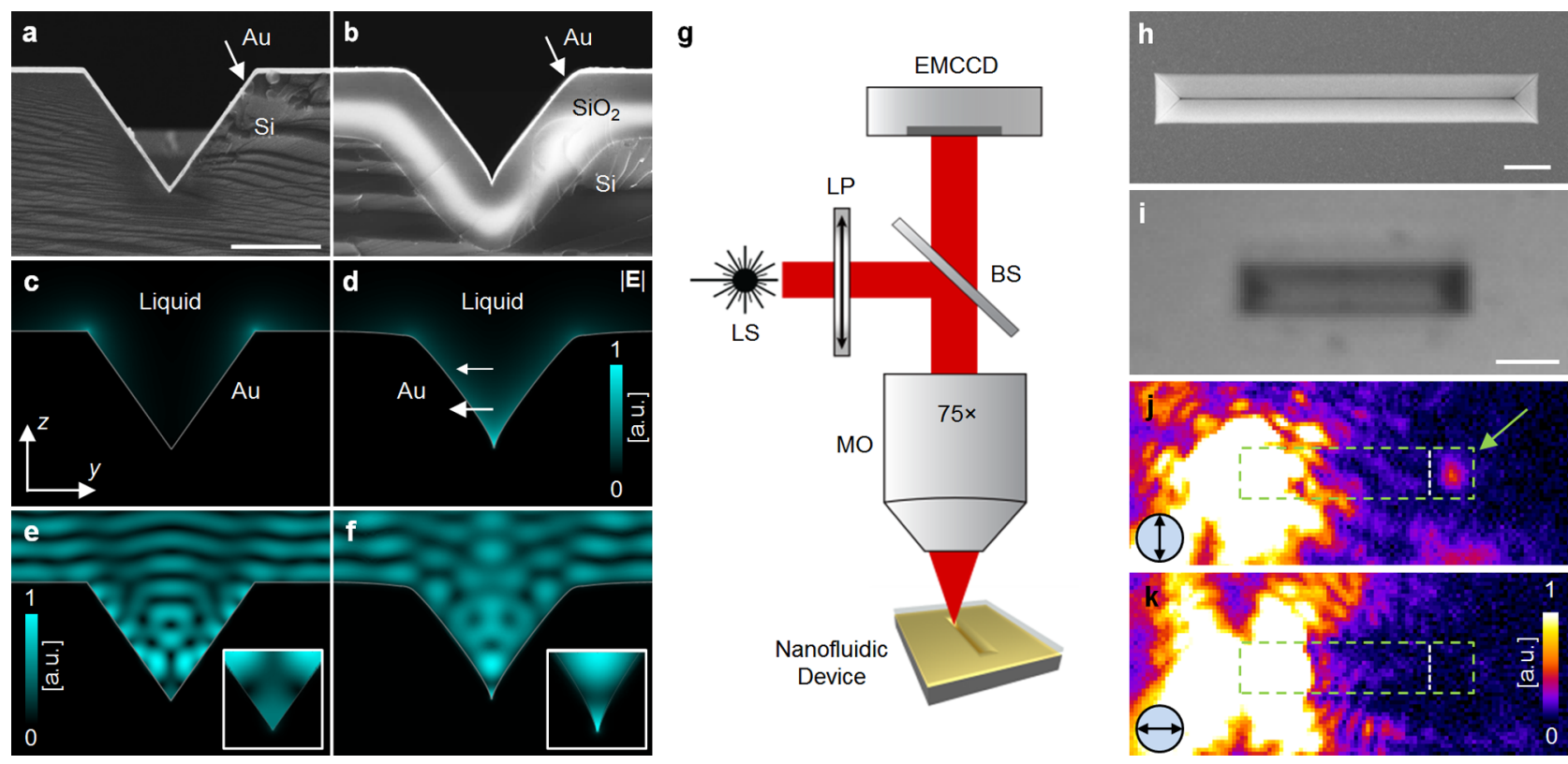

Figure 3. (a,b) SEM images of V-groove cross sections formed by initial crystallographic etching of $\langle 100\rangle$ planes in Si. The geometry of (a) is modified in (b) by a $750 \mathrm{~nm}$ thick thermally grown $\mathrm{SiO}_{2}$ layer on the etched $\mathrm{Si}$ prior to metallization. The scale bar is $1 \mu \mathrm{m}$. (c,d) Twodimensional FEM calculations of the plasmonic modes corresponding to the V-groove profiles of (a,b), respectively. (c) Undesired wedgelocated modes result from the geometry of $(a)$, whereas in $(d)$, the modification leads to a sufficiently sharpened vertex at the bottom of the waveguide to support CPP modes. The white arrows in (d) indicate an instantaneous electric field orientation. (e,f) Distributions of $|E|$ resulting from a planar wave (elliptical polarization) normally incident on the structures from above. The insets denote $0.5 \mu \mathrm{m} \times 0.5 \mu \mathrm{m}$ regions at the vertices. (g) Experimental setup for confirming CPP excitation. Light from a laser source $\left(\mathrm{LS}, \lambda_{0}=811 \mathrm{~nm}\right)$ passes through a linear polarizer (LP), reflects off from a beam splitter (BS), and is focused by a microscope objective (MO, $75 \times$ ) onto the device containing liquid-immersed V-grooves. The out-coupled radiation is detected by an EMCCD. (h) Top-view SEM image of a $\approx 30 \mu \mathrm{m}$ length V-groove tailored as in (b). The scale bar is $2 \mu \mathrm{m}$. (i) Bright-field image of a $\approx 10 \mu \mathrm{m}$ length V-groove. The scale bar is $2 \mu \mathrm{m}$. (j,k) Experimentally observed radiation out-coupled from the device of $(i)$. The circle insets denote the orientation of the incident electric field. The dashed green lines represent the border of the embedded V-groove structures. The area enclosed by the white and green dashed lines indicates the integration region for determining the relative out-coupling powers. The small spot of out-coupled light in ( $j$ ) (green arrow, right) is larger than that for the same region in $(\mathrm{k})$, indicating CPP excitation.

dimensions over a flat surface region. In Figure $2 \mathrm{e}$, the $\lambda$-DNA enters the vicinity of the V-groove trap $(1.28 \mu \mathrm{m}$ maximum groove depth plus $0.38 \mu \mathrm{m}$ slit height) and accelerates toward it due to entropic recoil. ${ }^{2,12}$ The DNA subsequently remains in the V-groove by entropic confinement. In contrast, Figure $2 f$ shows the negligible influence of the $\mathrm{V}$-groove on the motion of two fixed-shape nanospheres of $140 \mathrm{~nm}$ diameter. These nanospheres are around half the maximum size of fixed-shape objects that can freely enter the nanoslit region. Both particles move into the presented region from the left after the laser is turned on. Their trajectories are dominated by Brownian motion, including when passing over regions of strong thermal gradient caused by an incident beam of $61 \mathrm{~mW}$ focused on the V-groove nanomirror (red spot) as their radii lead to a small thermophoretic coefficient. ${ }^{32}$ This highlights the particular role of the entropic landscape formed by the embedded V-grooves, in which the trapping is exclusive to flexible molecules due to the difference of entropy for these molecules in the V-grooves compared to the slits. It is important to note that such a change of entropy does not occur for fixed-shape objects.

Tailoring and Confirmation of Plasmons. The photothermal transport of DNA in this work is induced by the losses of tailored plasmonic modes. The tailoring is achieved by modifying the V-groove profiles, initially defined by the crystallographic planes of $\mathrm{Si}$ (Figure 3a), with a thermally grown $750 \mathrm{~nm}$ thick $\mathrm{SiO}_{2}$ layer to sharpen the vertex (Figure $3 \mathrm{~b})$ and support desired CPPs. This modification is based on a similar reported process, ${ }^{41}$ here considering a liquid-cladding refractive index. Further details of the fabrication are in the Methods section and the Supporting Information. Figure 3c,d shows the 2D FEM-calculated modes ( $|E|)$ supported by the profiles of Figure $3 a, b$, respectively, indicating the prevalence of wedge plasmon polaritons (WPPs) in Figure $3 \mathrm{c}$ (propagation length, $\left.L_{\mathrm{WPP}}=14.6 \mu \mathrm{m}\right)$ compared to the single, central maximum CPP mode $\left(L_{\mathrm{CPP}}=10.9 \mu \mathrm{m}\right)$ of Figure $3 \mathrm{~d}$. The white arrows in Figure $3 \mathrm{~d}$ denote the instantaneous electric field orientation, which tends across the $\mathrm{V}$-groove. The absence of $\mathrm{Si}$ and $\mathrm{SiO}_{2}$ in these mode calculations simplifies the calculation and avoids unwanted computational artifacts. This is valid due to the large absorption of the gold layer that isolates the electric field of the top interface (metal-liquid) from the interface beneath $\left(\right.$ metal- $\left.\mathrm{SiO}_{2}\right){ }^{41}$ The distributions of $|\mathrm{E}|$ in Figure $3 \mathrm{e}, \mathrm{f}$ result from a planar wave (elliptical polarization) normally incident on the structures. The insets denote $0.5 \mu \mathrm{m}$ $\times 0.5 \mu \mathrm{m}$ regions at the vertices, highlighting that the tailored $\mathrm{V}$-groove also supports gap plasmons which focus toward the $\mathrm{V}$-groove vertex. The separate local maxima of $\partial T / \partial x$ in Figure le slightly along the waveguide and at the nanomirror additionally indicate that a combination of $\mathrm{CPPs}^{41}$ and focusing gap plasmons, ${ }^{42}$ respectively, is present.

The excitation of CPPs is experimentally confirmed by assessing the polarization anisotropy ${ }^{41}$ of out-coupled light using the experimental setup of Figure 3g. Direct observation of gap plasmons in our device configuration is beyond the scope 
of this work. Figure $3 \mathrm{~h}$ is a SEM image of $\mathrm{a} \approx 30 \mu \mathrm{m}$ length $\mathrm{V}$ groove tailored as in Figure $3 \mathrm{~b}$, and Figure $3 \mathrm{i}$ is a bright-field image of a similar but $\approx 10 \mu \mathrm{m}$ length $\mathrm{V}$-groove used in experiments. The panels of Figure 3j,k show the radiation detected by the electron multiplying charge-coupled device (EMCCD) out-coupled from the nanomirror. The large white regions of Figure $3 \mathrm{j}, \mathrm{k}$ are scattered components from the incident light; the intensity scales are co-normalized. The circle insets denote the orientation of the incident electric field, which in Figure $3 j$ matches that of the CPP in Figure 3d. The small spot in Figure $3 j$ is out-coupled light from the $\mathrm{V}$-groove nanomirror, for which the intensity integrated over the region enclosed by the white and green dashed lines (corrected for dark current noise) is 5.7 times greater than for the same in Figure $3 \mathrm{k}$. This result supports the assumption that the excitation of plasmonic modes occurs via normal illumination of the nanofluidic nanomirrors.

Plasmonic Heating. In order to assess the heat generated by the excited plasmons, we first map the temperature in the Vgrooves experimentally by introducing a solution of $[\mathrm{Ru}-$ $\left.(\mathrm{bpy})_{3}\right]^{2+}$ to the device, which exhibits a temperaturedependent fluorescent emission intensity ${ }^{33,43}$ (Figure 4a).
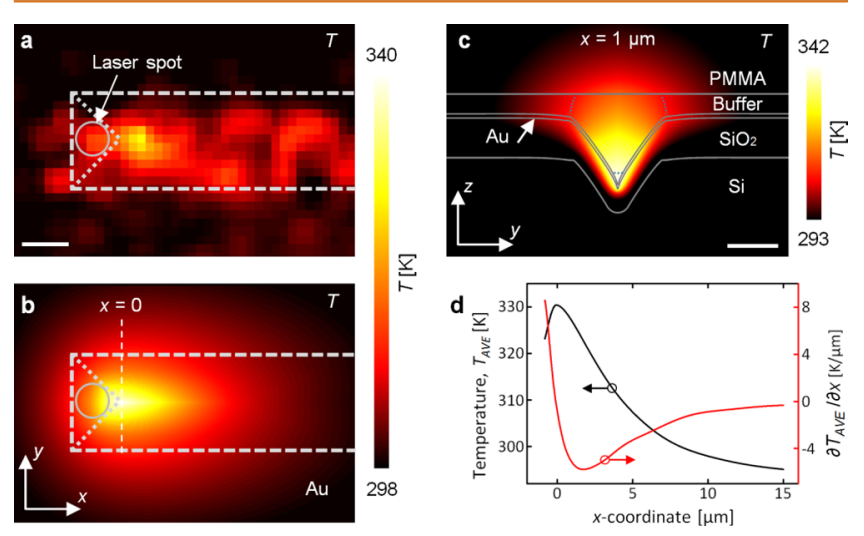

Figure 4. (a) Experimental mapping of the temperature inside a nanofluidics-integrated $\mathrm{V}$-groove for $30 \mathrm{~mW}$ incident power on the nanomirror using a solution of temperature-dependent fluorescing $\left[\mathrm{Ru}(\mathrm{bpy})_{3}\right]^{2+}$. The dashed gray line represents the $\mathrm{V}$-groove, and the solid gray circle is the location of the laser spot (similar conditions as Figure 1). The scale bar is $1 \mu \mathrm{m}$. (b) Threedimensional FEM-calculated temperature averaged through $z$ (liquid region only) of the system corresponding to (a). (c) Calculated temperature at the $x=1 \mu \mathrm{m}$ plane. The scale bar is 1 $\mu \mathrm{m}$. (d) Calculated estimate of the cross-sectional average temperature, $T_{\text {ave }}$ (black line), and its gradient, $\partial T_{\text {ave }} / \partial x$ (red line), experienced by a $\lambda$-DNA molecule along the V-groove axis.

The observed temperature for $30 \mathrm{~mW}$ of incident power on the nanomirror corresponds to the average temperature throughout the depth of the liquid region projected onto the 2D $x y$-plane. Misalignment of the acquired temperature profile with respect to the groove is attributed to a slight tilt of the device with respect to the imaging plane. Further details of the temperature mapping are in the Methods section and the Supporting Information. Figure $4 \mathrm{~b}$ shows the corresponding 3D FEM-calculated temperature averaged over the $z$-direction of the liquid region. Further details of the temperature mapping and numerical modeling are in the Methods section and the Supporting Information. The resulting directivity of the temperature distribution along the $\mathrm{V}$-groove stems from the dissipation of propagating CPPs and is in agreement with the measurement of Figure 4a. It is interesting to note that the maximum temperature occurs along the waveguide, displaced from the incident beam maximum, due to the excitation of CPPs via the angled mirror. Figure $4 \mathrm{c}$ is the calculated temperature along the $x=1 \mu \mathrm{m}$ plane. The temperature increase occurs maximally at the bottom of the V-groove, with a significant portion extending up through the liquid. The dashed lines indicate the region where a DNA molecule confined by the $\mathrm{V}$-groove may extend. To estimate the photothermal effect experienced by a $\lambda$-DNA molecule in the system, the crosssectional average temperature, $T_{\text {ave }}$ (black line), and its gradient, $\partial T_{\text {ave }} / \partial x$ (red line), are plotted in Figure $4 \mathrm{~d}$ versus the groove $x$-axis coordinate. The temperature gradient determines the thermophoretic force experienced by a $\lambda$ DNA molecule in the V-groove. It is worth remarking that the temperature at which denaturation of DNA begins $(\approx 338 \mathrm{~K})^{44}$ is higher than the temperature experienced by such a molecule at this input power (e.g., $5 \mu \mathrm{m}$ from the mirror the average temperature is $\approx 310 \mathrm{~K}$ ). Such DNA melting, in which the molecule would become single-stranded and coil into smaller gyration radii, was not observed in our experiments. For higher powers, one could propel the DNA starting further along the groove to avoid DNA melting temperatures.

As mentioned earlier, the calculated coupling efficiency from elliptically polarized incident light to heat-generating plasmons is found to be $\eta=20.4 \%$, which represents the combined contributions from CPPs and gap plasmons, that is, $\eta=\eta_{\mathrm{CPP}}+$ $\eta_{\mathrm{GP}}$, with both right-hand terms polarization-dependent. From 3D FEM simulations, we determine the coupling efficiencies into liquid-cladding CPPs for the polarizations elliptical (experiment) $\eta_{\mathrm{CPP} \text {,ellip }} \approx 6 \%$, transverse electric (TE) (perpendicular to the V-groove axis) $\eta_{\mathrm{CPP}, \mathrm{TE}} \approx 13 \%$, and long (parallel to the V-groove axis) $\eta_{\mathrm{CPP}, \text { long }} \approx 2 \%$. The coupling efficiencies to gap plasmons, more challenging to isolate in the model, should be at a maximum for TE polarization and have a moderate dependence on the incident light polarization due to the structure of the nanomirror. Accordingly, the heat generation would be most efficient for TE-polarized incident light (not possible in the existing setup; see Methods). Based on the calculated heating, we estimate $\eta_{\mathrm{GP} \text {,ellip }} \approx 14 \%$.

The plasmonic heating dependence on the properties of the incident laser beam (location, extremities of polarization) and the presence of the gold layer is presented in the numerically calculated temperature profiles of Figure 5. Here, the inset illustrations denote the location and polarization of the incident beam, for which the power is $10 \mathrm{~mW}$ in all panels, and the temperature color scale is co-normalized. In Figure 5a,b, the laser is focused on the nanomirror, with the polarization in Figure 5a corresponding to efficient excitation of CPPs which leads to heat generation extending along the waveguide. This contrasts to the case of Figure $5 \mathrm{~b}$, which generates heat at a reduced efficiency and extent from the mirror. In Figures $5 c$,d, the laser is focused midway along the groove, with the polarization in Figure $5 \mathrm{c}$, d corresponding to relatively efficient and inefficient excitation of focusing gap plasmons, respectively. This temperature increase does not exhibit the directed extension caused by propagating CPPs in Figure 5a but is large compared to that of Figure $5 \mathrm{~d}$ and nevertheless suggests that a tracking-mode configuration would be possible to move entropy-trapped molecules over as-desired distances along the waveguide. In Figure 5e,f, the laser is focused on the nanomirror but without the presence of the gold layer to support plasmons. Here, both polarizations lead to maximum 

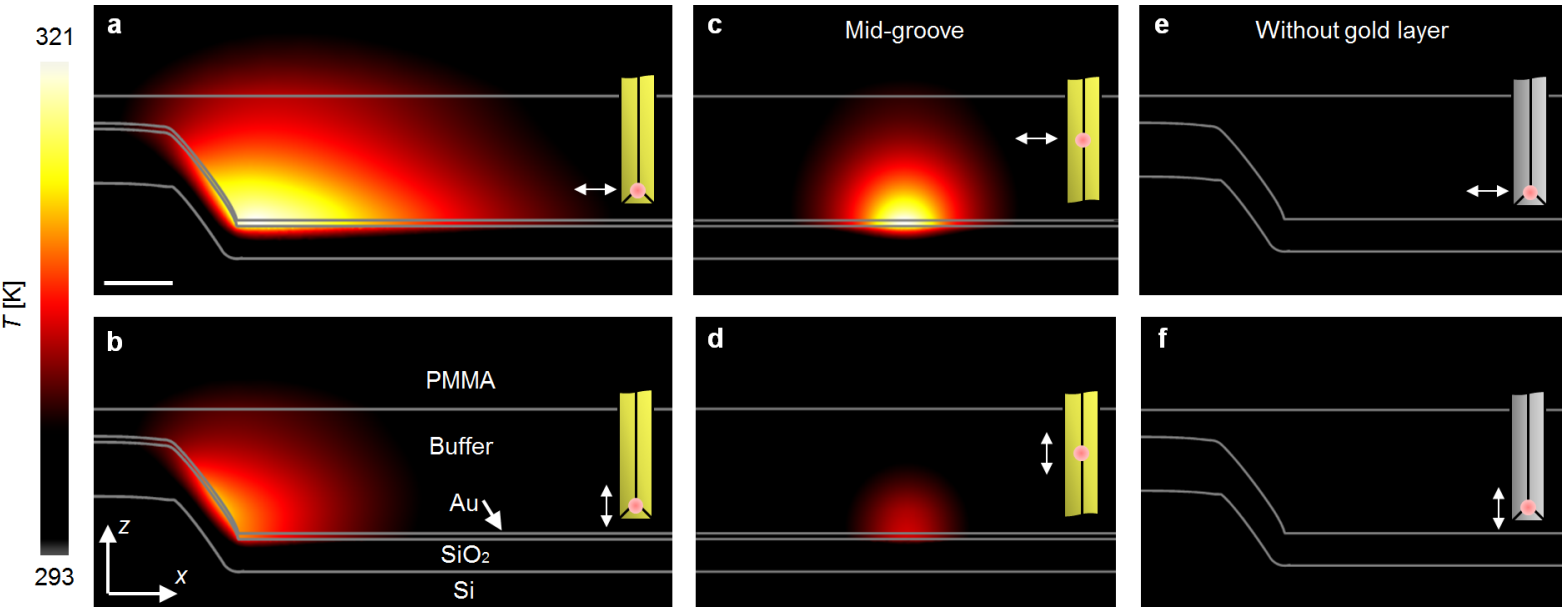

Figure 5. Calculated temperature profiles caused by laser light focused on the V-grooves. The insets represent the location and polarization of the incident laser beam. The incident power is $10 \mathrm{~mW}$ in all cases, and the thermal color map on the left corresponds to all panels of the figure. (a) Laser light is focused on the nanomirror with its polarization oriented perpendicular to the V-groove axis. The scale bar is $1 \mu \mathrm{m}$. (b) Similar plot to (a) but with the laser light polarized parallel to the groove axis. (c,d) Laser light is focused on the V-groove but away from the nanomirror for perpendicular (c) and parallel (d) incident polarizations. (e,f) Laser light is focused on the nanomirror but without the gold layer present for perpendicular $(e)$ and parallel $(f)$ incident polarizations.
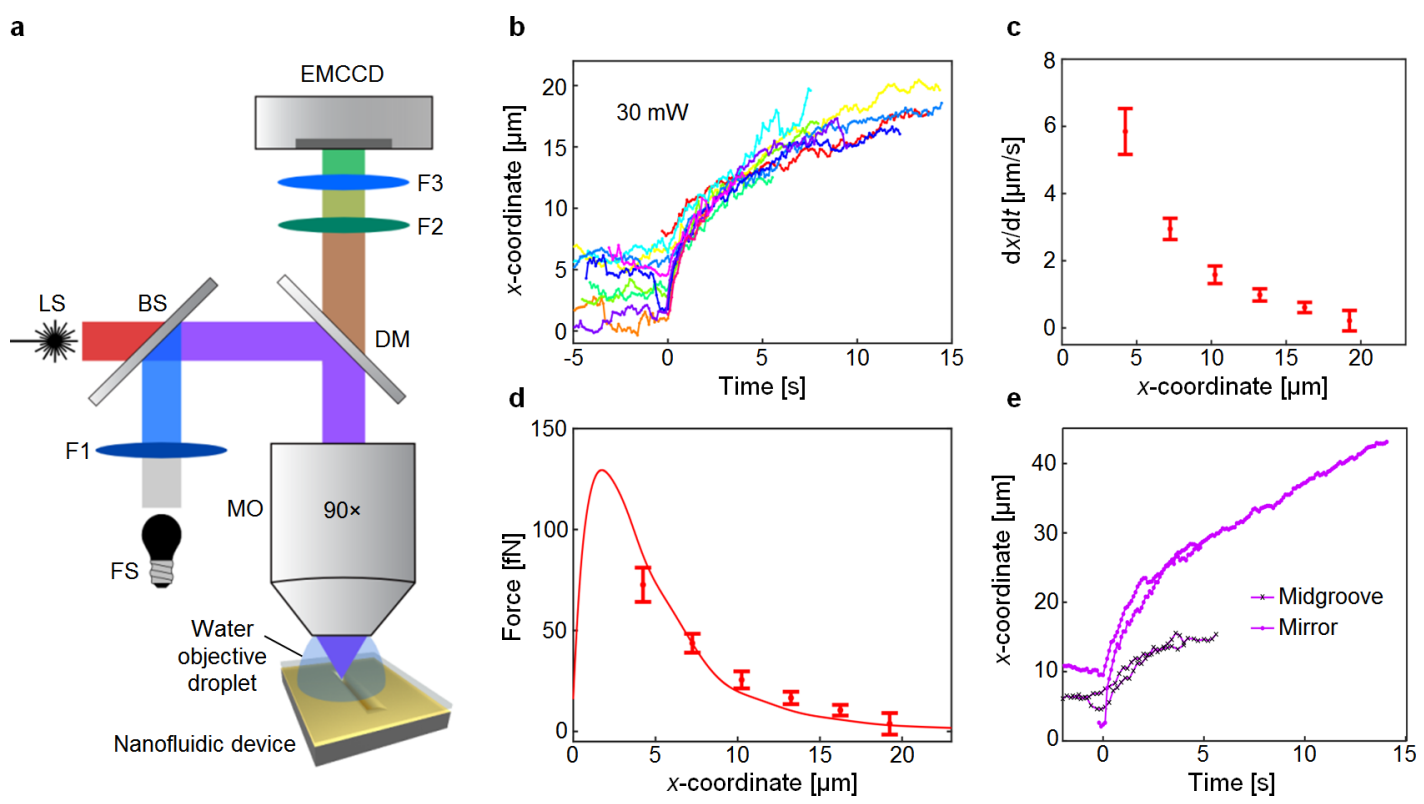

Figure 6. (a) Experimental setup for simultaneously exciting plasmons and imaging DNA. Light from a fluorescent excitation source (FS) passes through an excitation filter $(F 1,460-500 \mathrm{~nm})$ and combines with light from the LS at a BS, which is then directed to a dichroic mirror (DM). The DM reflects the light through a water immersion MO $(90 \times)$ onto the device, with the out-coupled radiation returning back through the MO. Reflected laser light is subsequently blocked while the fluorescent emission passes through the DM, an emission pass filter $(F 2,520-570 \mathrm{~nm}$ ), and a short-wavelength pass filter (F3) before detection by the EMCCD (with gain). (b) Position of $12 \lambda$-DNA versus time for an incident laser power of $30 \mathrm{~mW}$. The laser is focused on the nanomirror (terminating at $x=0$ ) and switched on at time $t=0$. (c) Conditional velocity $\langle\mathrm{d} x / \mathrm{d} t\rangle_{x}$ versus position for the trajectories in (b) obtained by bin averaging the instantaneous velocities $\mathrm{d} x / \mathrm{d} t$ (bin widths equal to $3 \mu \mathrm{m})$. (d) Extracted force and a fit (red line) to the thermophoretic force $F_{\text {therm }}(x)=-S_{\mathrm{T}} \partial T / \partial x$ with the temperature gradient $\partial T / \partial x(x)$ from the model calculation (Figure 4d). (e) Comparison of the motion of the DNA for the laser focused at the nanomirror (filled magenta circles) and midway along the groove (black crosses), each for a laser power of $180 \mathrm{~mW}$. The midway data are offset so $x=0$ corresponds to the center of the laser beam. All error bars are standard errors of the means.

temperature increases of less than several degrees kelvin as the water and glass materials have little absorption at our laser wavelength. The dependence of the resulting temperature on the incident laser beam location and polarization, plus the presence of the gold layer, highlights the role of plasmons for device heating.
Photothermal Transport. Combining the entropic trapping with the plasmon-induced heat generation, we study the photothermal transport of $\lambda$-DNA in the V-grooves with the experimental setup shown in Figure 6a (see further details in the Methods section). When the laser is off, the DNA molecules diffuse in the $\mathrm{V}$-groove with no sign of drift (see section 1 of the Supporting Information). Figure $6 \mathrm{~b}$ shows the 
a

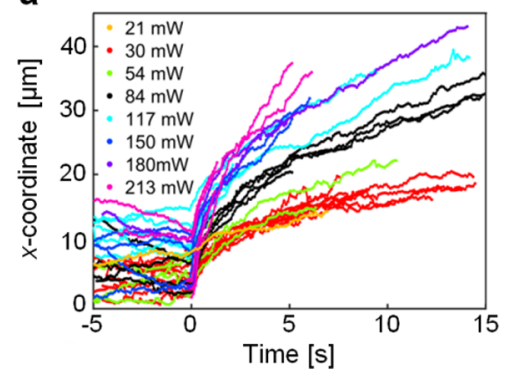

b

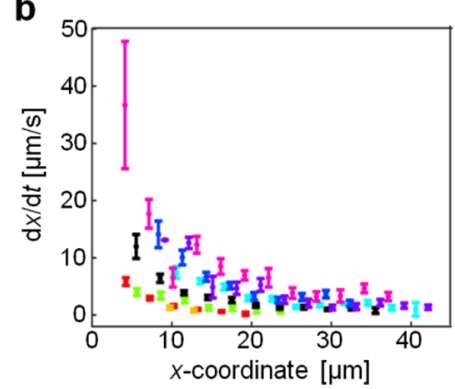

C

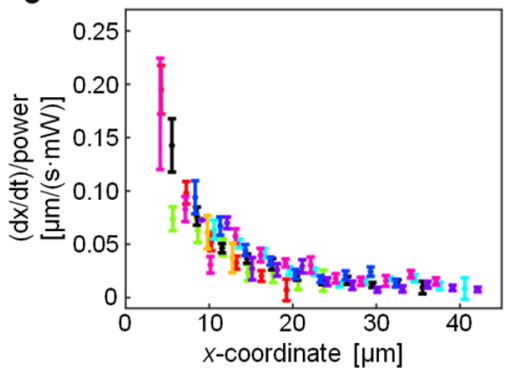

Figure 7. (a) Position of the DNA versus time for eight different laser powers. The laser is focused on the nanomirror (terminating at $x=0$ ) and switched on at time $t=0$. (b) Conditional velocities $\langle\mathrm{d} x / \mathrm{d} t\rangle_{x}$ versus position for the trajectories in (a) obtained by bin averaging the instantaneous velocity $\mathrm{d} x / \mathrm{d} t$ (bin widths equal to $3 \mu \mathrm{m}$ ). (c) Conditional velocities scaled with laser power.

positions of 12 separate $\lambda$-DNA molecules propagating along the V-groove versus time, where the laser is switched on at time $t=0$ (for $30 \mathrm{~mW}$ incident laser power). The laser causes an abrupt onset of directed motion.

To quantify the forces acting on the DNA, we model the motion of the DNA molecules with Newton's second law, which in the overdamped limit $\left|m \mathrm{~d}^{2} x / \mathrm{d} t^{2}\right| \ll|\gamma \mathrm{d} x / \mathrm{d} t|$ ( $m$ is the mass of a $\lambda$-DNA molecule) reduces to

$$
\frac{\mathrm{d} x}{\mathrm{~d} t}=\frac{F_{\text {ext }}(x)}{\gamma}+\sqrt{2 D} \xi(t)
$$

Here, $F_{\text {ext }}(x)$ is the position-dependent external force we want to determine, and $\gamma$ is the friction coefficient for DNA in the Vgroove. The last term is a stochastic (Brownian) force acting on the DNA due to the interaction of DNA with the surrounding water molecules. The diffusion constant is $D=k_{\mathrm{B}} T / \gamma$, and $\xi(t)$ is a Gaussian white noise with zero mean and unit variance $\left[\langle\xi(t)\rangle=0\right.$ and $\left\langle\xi(t) \xi\left(t^{\prime}\right)\right\rangle=\delta\left(t-t^{\prime}\right)$ for all $t, t^{\prime}$, where $\delta(t)$ is a Dirac delta function and $\langle\cdot\rangle$ denotes expected value]. The diffusion constant, and thus the friction coefficient, $\gamma$, can be determined from the diffusive motion of the DNA with the laser off (see section 1 of the Supporting Information). We obtain $\gamma=18 \mathrm{fN} \mathrm{s} / \mu \mathrm{m}$; approximately two to three times its value in bulk.

The external force acting on the DNA at position $x$ can be extracted from the velocities by the conditional average ${ }^{45,46}$

$$
\left\langle\frac{\mathrm{d} x}{\mathrm{~d} t}\right\rangle_{x}=\frac{F_{\text {ext }}(x)}{\gamma(x)}
$$

where $\langle\cdot\rangle_{x}$ is the average given the position $x$. We find the conditional average in eq 5 from the experimental data in Figure $6 \mathrm{~b}$ by calculating the velocity versus the $x$-coordinate and then averaging over bins with a width of $3 \mu \mathrm{m}$. The results are shown in Figure 6c, left axis. The external force, $F_{\text {ext }}(x)=$ $\gamma(x)\langle\mathrm{d} x / \mathrm{d} t\rangle_{x}$, is shown in Figure 6d. We here write $\gamma(x)$ due to the positional change in viscosity as a function of the local temperature, $T(x)$.

Figure $6 \mathrm{~d}$ also shows a fit to the derived thermophoretic force using the calculated temperature gradient of Figure $4 \mathrm{~d}$. For this laser power, we assume that the external force is dominated by thermophoresis, that is, $F_{\text {ext }}(x)=F_{\text {therm }}(x)$, where $F_{\text {therm }}(x)=-k_{\mathrm{B}} T S_{\mathrm{T}} \partial T / \partial x$. Here, $S_{\mathrm{T}}$ is the Soret coefficient, and $T(x)$ is the position-dependent temperature profile. ${ }^{32}$ The fitted value of the Soret coefficient is $S_{\mathrm{T}}=(5.5 \pm 0.6) \cdot \mathrm{K}^{-1}$, roughly a factor of 4 larger than a previous measured bulk value for $\lambda$-DNA. ${ }^{32}$ Possibly, this difference is caused by the V-groove structure's influence on the heat flow around the DNA, although the Soret coefficient depends critically on parameters not provided ${ }^{32}$ such as the base temperature and the Debye screening length in the solvent, so one should be cautious when comparing values. A $\chi^{2}$ goodness-of-fit test returns a $p$ value of 0.0275 , slightly less than the standard $5 \%$ criterion, so the thermophoretic model may not capture all features in the data, which might also explain the large $S_{\mathrm{T}}$ value. Even so, when held against a previous photothermal-based scheme that relied on a dye-doped polymer absorber layer, ${ }^{33}$ our device here provides approximately double the propagation velocity for a similar laser power and additionally benefits from extended motion far from the incident beam as well as entropic self-alignment.

We do not observe an optical gradient force attracting the DNA back toward the V-groove mirror in our experiments. We attribute this to the shorter characteristic length scales of the electric field gradients compared to the force profiles caused by heating. Consider that the force may occur from two possible sources in our configuration: (1) the decay of propagating CPP modes or (2) the evanescent tail of the beam. For (1), approximate calculations indicate that this is several orders of magnitude less than the photothermal effect even for modest distances (several microns) from the nanomirror (see section 5 of the Supporting Information). For (2), this value becomes vanishingly small already within a micron from the beam center. Similar to (1), a downward force toward the bottom of the Vgroove via the electric field gradient of the CPP mode cross section is not observed; we expect it would be overcome by an opposing upward force caused by the vertical temperature gradient, $\partial T / \partial z$.

Figure $6 \mathrm{e}$ demonstrates the importance of utilizing the mirrors terminating the $\mathrm{V}$-grooves and hence the efficient excitation of CPPs by directly comparing the DNA motion when the laser is focused at the mirror with midway along the $\mathrm{V}$-groove. The extent of the motion is reduced when the laser is focused away from the mirror, indicating that in this case only the gap plasmons (and material-based absorption) are present to induce the temperature gradient. We note, however, that this result confirms the possibility to use the photothermal effect to transport entropy-trapped molecules over arbitrary distances via tracking methods, in which the heat may be being generated by the laser at any location along a waveguide route.

The positions of DNA molecules along the $\mathrm{V}$-groove versus time are presented in Figure $7 \mathrm{a}$ for eight different incident laser powers $(21-213 \mathrm{~mW})$. Figure $7 \mathrm{~b}$ shows the corresponding conditional velocities versus position for the trajectories in Figure $7 \mathrm{a}$. The maximum measured velocities for each incident power are found to be $\approx 0.2 \mu \mathrm{m} \cdot \mathrm{s}^{-1} \cdot \mathrm{mW}^{-1}$, whereas the extent of the movement reaches $\approx 0.5 \mu \mathrm{m} \cdot \mathrm{mW}^{-1}$. Thermophoresis is 
therefore not suitable for fast transport over large distances when compared to hydrodynamic pumping or electrophoresis. However, photothermal transport does offer particularly high localizability and flexibility, ${ }^{33}$ which enable it to be incorporated with other chip-based functionalities such as, in our case, entropic trapping. To assess the power scaling, Figure 7c plots the power-normalized values of data from Figure $7 \mathrm{~b}$. The scaled data points fall almost on a common curve, demonstrating that the external force is proportional to the applied laser power. The exception is far from the mirror, where for lower laser powers $(\leq 54 \mathrm{~mW})$ the velocity drops to zero before $x=20 \mu \mathrm{m}$, while for higher powers $(\geq 84 \mathrm{~mW})$ the velocity tends to a constant level in our measurement range.

The DNA motion caused by lower laser powers may be explained mostly by thermophoresis, but for higher powers, we note that the gradient along the waveguide $\partial T(x) / \partial x$ of the calculated temperature profile $T(x)$ decays faster than the measured velocities. In this case, we rule out light pressure (conversion of scattering and absorption to kinetic energy) due to the rapid rates of free space light divergence and plasmonic dissipation and instead speculate that the system undergoes nonlinear processes concerning, for example, thermo-optic coefficients, convective flow, the Peltier-Seebeck effect, or a phase change (bubble formation) of the buffer solution. An accurate description of the physics involved in the system at higher powers thus presents itself as an interesting challenge for future work, for example, by considering the velocity scaling with input power, as it may lead to greatly extended motion of DNA far from an incident laser beam and play a generally crucial role in devices that involve plasmonic heating to transport biomolecules throughout nanofluidic networks.

\section{CONCLUSIONS}

In summary, we have demonstrated the fusion of nanoconfined polymer dynamics with photothermal manipulation to achieve photothermal transport of entropy-guided DNA molecules using nanofluidics-integrated plasmonic V-grooves. First, we verified the entropic confinement of $\lambda$-DNA in the embedded $\mathrm{V}$-grooves and used a transverse flow for controlled loading of the molecules to lines of interest. By contrast, fixed-shape nanospheres with a $140 \mathrm{~nm}$ diameter were neither trapped by the V-grooves nor significantly influenced by an induced heat profile. Second, based on the results of 2D numerical simulations, we tailored the liquid-cladding V-grooves with a thermal oxidation step in order to support CPP modes and focusing gap plasmons, from which the excitation of CPPs in our device was confirmed by polarization anisotropy experiments. Third, three-dimensional simulations coupling the electromagnetic solution to a heat transfer model allowed temperature profiles to be determined that agreed with heat mapping experiments obtained using a temperature-dependent dye solution. The in-coupling efficiency of light to heatgenerating plasmons, found to be $\eta=20.4 \%$ (plus $4.1 \%$ material-based absorption), led to the generation of directed heat flow along the waveguide due to the presence of CPPs. Simulations presenting the importance of coupling via the nanomirror and the presence of the gold film highlighted the role of plasmonic heating in the system. Fourth, fitting the position-dependent, experimentally determined forces extracted from DNA trajectories for $30 \mathrm{~mW}$ laser power to a calculated temperature gradient profile, we obtained a Soret coefficient 4 times larger than a previous measurement in bulk. A direct comparison of values is difficult because this value depends critically on parameters not provided such as base temperature and the Debye screening length in the solvent. Nevertheless, most of the transport behavior can be explained by thermophoresis, especially at our lower range of input powers, while the behavior at higher input powers indicates additional physics yet to be elucidated that may offer a pathway to realize yet larger extensions of transport away from the input beam. Finally, we quantified the practical performance of our device, which can move $\lambda$-DNA with velocities of $\approx 0.2 \mu \mathrm{m} \cdot \mathrm{s}^{-1} \cdot \mathrm{mW}^{-1}$ and provide transport over distances up to $\approx 0.5 \mu \mathrm{m} \cdot \mathrm{mW}^{-1}$ away from the input beam.

Principally, our light-driven actuator overcomes the challenges of nanoscale interaction volumes by means of the entropic self-alignment in the dual-functional plasmonic surface, a technique that may be applied generally to single, flexible molecules. Looking forward, our platform enables a set of capabilities to optically handle individual free DNA molecules via the convenience of readily available microscope systems, which could forego the need for tracking methods or, conversely, be implemented as an extensive routing technique that relies on entropic self-alignment to efficiently adhere to specified microscale paths. Our work may lead to a range of exciting possibilities, such as site-specific sensing and/or reagent exchange, molecular filtering from mixtures, or analysis of biochemistry on individual molecules. Moreover, these prospects are especially promising as our devices may be fabricated exclusively by wafer-scale methods that are inherently suited to mass production and large-scale parallelization.

\section{METHODS}

Chip Fabrication. A Si substrate with a $200 \mathrm{~nm}$ thick $\mathrm{SiO}_{2}$ layer was patterned by UV lithography and reactive ion etching to define the perimeter of the V-groove devices (initial widths were $2.2 \mu \mathrm{m}$, and lengths were $10,20,30,50$, and $115 \mu \mathrm{m})$. The V-grooves and nanomirrors were formed by anisotropic wet etching of the exposed $\mathrm{Si}$ in a potassium hydroxide bath at $80^{\circ} \mathrm{C}$, resulting in smooth $\langle 111\rangle \mathrm{V}$ groove sidewalls and nanomirrors with crystal-determined angles of $55^{\circ}$ from the surface plane. The remaining $\mathrm{SiO}_{2}$ was removed by etching in a hydrofluoric acid bath. Tailoring of the $\mathrm{V}$-shape geometry was achieved by thermal wet oxidation of the $\mathrm{Si} \mathrm{V}$-grooves ${ }^{4 \mathrm{P}}$ at 1150 ${ }^{\circ} \mathrm{C}$ for $60 \mathrm{~min}$, resulting in a $750 \mathrm{~nm}$ thick $\mathrm{SiO}_{2}$ layer at flat sections of the substrate. The V-groove widths were approximately $1.9 \mu \mathrm{m}$ after the thermal oxidation step. A $2.2 \mu$ m thick UV resist was UV-patterned to define select regions for metal deposition. The gold film was deposited by electron beam evaporation: first, a $6 \mathrm{~nm}$ layer of chromium to promote adhesion to the $\mathrm{SiO}_{2}$ layer before a $70 \mathrm{~nm}$ layer of gold. The remaining UV resist was dissolved in acetone and ultrasonic agitation, removing most of the metal from the wafer in order to improve the adhesion of an SU-8 polymer layer to the $\mathrm{SiO}_{2}$ in the following steps. A $0.38 \mu \mathrm{m}$ thick SU-8 layer was applied and UVpatterned to define the nanoslit fluid channels. An array of $1 \mathrm{~mm}$ fluid inlet-outlet ports were milled through the wafer by laser micromachining. A separate $\mathrm{Si}$ substrate with $200 \mathrm{~nm}$ thick $\mathrm{SiO}_{2}$ layer was patterned by UV lithography and reactive ion etching to form a stamp with $4 \mu \mathrm{m}$ height protrusions. The stamp was imprinted onto a glass wafer supporting a deformable PMMA layer, defining the access microchannels. The two wafers with SU-8 and PMMA were optically aligned and thermally bonded at $115{ }^{\circ} \mathrm{C}$ for 60 min with $2000 \mathrm{~N}$ to form the sealed device. ${ }^{47} \mathrm{~A}$ fabrication schematic and optical images of resulting devices are in section 3 of the Supporting Information.

Microscope Facility. The emission from a monochromatic continuous-wave fiber-coupled diode laser at a free space wavelength of $\lambda_{0}=811 \mathrm{~nm}$ [full width at half-maximum $(\mathrm{fwhm})=0.3 \mathrm{~nm}$ ] was guided through a connector module (Ti-PAU, Nikon) into the optical path of an inverted microscope (Nikon Eclipse TE2000-U). The chuck for the plasmonic-nanofluidic chips enabled the solution to be pipetted 
to the device inlet ports and air hoses to be connected to Luer pressure fittings for controlled fluid flow. The chuck was fixed to a 2-axis piezoelectric stage (to optimize in-coupling) installed on the 3-axis stage of the microscope. The laser was focused on the sample through an objective, for which the microscope provided an additional $1.5 \times$ magnification. The laser did not pass through a polarizer during experiments with DNA to avoid the reduction of fluorophore excitation light; accordingly, the simulations and experiments consider the elliptical polarization as measured to illuminate the sample. The light from the sample was imaged through the same objective using a $-70{ }^{\circ} \mathrm{C}$ cooled vacuum-sealed linear EMCCD (Photometrics) with $512 \times 512$ pixel array $(16 \mu \mathrm{m} \times 16 \mu \mathrm{m})$.

Optical Characterization. The polarization of the incident laser light on the sample was controlled by a linear polarizer installed prior to a beam splitter. A $50 \times$ magnification, 0.8 numerical aperture (NA), and $1 \mathrm{~mm}$ working distance (WD) air objective were used. The gain of the EMCCD was set to 0 to maximize the dynamic range of the image and to maintain a linear response of the charge coupling. The focused laser beam had a spot size fwhm of $1.1 \mu \mathrm{m}$, measured from an image of the light reflected from a flat gold surface. The relative coupling efficiencies of the polarization states were obtained by integrating the pixel values over regions wholly containing the out-coupled signal. To ensure the same relative focal plane for the two incident polarization states, the in-coupling was first $x y z$-maximized for the case of large outcoupling efficiency and then translated aside $5 \mu \mathrm{m}$ to obtain the spot size on the adjacent flat section. After rotating the polarization, the procedure was reversed, first setting the same spot size $5 \mu \mathrm{m}$ adjacent to the V-groove before aligning the in-coupling. ${ }^{41}$

DNA and Nanoparticle Imaging. The nanofluidic device was connected through microchannels of $4 \mu \mathrm{m}$ depth between the inlet ports and the nanofluidic region. The $\lambda$-DNA, of $12 \mathrm{ng} / \mu \mathrm{L}$ concentration, was pipetted into an inlet port in a buffer solution [96.5\% $0.5 \times$ degassed Tris-borate EDTA (TBE), 3\% 2-mercaptoethanol, $0.5 \%$ Triton X-100]. The mixture of nanospheres (polystyrene) was diluted to a concentration of $\sim 500 \mathrm{ng} / \mu \mathrm{L}$ in a separate buffer solution ( $99.5 \% 0.5 \times \mathrm{TBE}, 0.5 \%$ Triton X-100). The objective (water immersion) had $60 \times$ magnification, $1.0 \mathrm{NA}$, and $2 \mathrm{~mm}$ WD. The linear polarizer from the previous experiments was removed to maintain sufficient fluorescent excitation light for the imaging of DNA and nanoparticles. For thermophoresis experiments, the polarization of the incident fiber-coupled laser on the sample was elliptical with a ratio of 2:1 more power in the orientation parallel to the groove axis. The laser light joined the optical path of the fluorescent excitation source (Prior Lumen $200 \mathrm{~W}$ ) using a beam splitting mirror in the connector module. A dichroic mirror (Chroma Technology, ZT488/561) permitted excitation and laser light to illuminate the sample while favoring fluorescent emission to pass to the EMCCD (with gain). Additional emission and short-wavelength pass filters prior to the EMCCD cleaned the emission signal further. The spectra of the optical components for these experiments can be found in section 4 of the Supporting Information. The DNA and nanoparticles were tracked using the ImageJ plug-in MOSAIC.

Temperature Mapping. A modified version of the DNA imaging setup was installed, here using a $50 \times$ magnification, $0.8 \mathrm{NA}, 1 \mathrm{~mm} \mathrm{WD}$ air objective and an emission filter with pass range of $605-655 \mathrm{~nm}$. A solution of $30 \mathrm{mM}$ ruthenium $\left[\mathrm{Ru}(\mathrm{bpy})_{3}\right]^{3+}$ in Milli-Q water was introduced to the device. To find a profile of ruthenium's temperaturedependent fluorescent emission intensity, a cartridge heater with an integrated K-type thermocouple was used to heat the sample. The fluorescence intensity was measured in the microchannels for intervals increasing $5{ }^{\circ} \mathrm{C}$ from 25 to $65{ }^{\circ} \mathrm{C}$, from which the dye's temperature dependence curve was found to closely match $I(T)=A_{0} \exp (-T /$ 18.6) $+I_{0}$. For the V-groove embedded in the nanoslit, calibration maps at 25 and $65{ }^{\circ} \mathrm{C}$ were taken such that each pixel's temperature response could be assessed by solving simultaneously for $A_{0}$ and $I_{0}$ to address variations of fluid depth. After flushing fresh dye through the imaging region to avoid hysteresis and bleaching effects, we recorded a measurement with the laser incident on the mirror. The measurements consisted of image stacks of 25 -frame averages. The resulting data were processed with a $3 \times 3$ pixel moving window average and a userdefined SMOOTHN function in MATLAB with an $S$ parameter of 0.1 .

Numerical Simulations. The modeling of V-grooves via FEM in COMSOL was performed by first generating cross-section profiles from process simulations in ATHENA. The tailored V-groove profiles had $200 \mathrm{~nm}$ curvature radii rounding introduced at the wedge locations according to the SEM image of Figure 3b; for the unoxidized $\mathrm{V}$-grooves, this rounding was set to $15 \mathrm{~nm}$. The $\mathrm{V}$-groove vertices were rounded with a $5 \mathrm{~nm}$ radius corresponding to previous work. ${ }^{41}$ The refractive index at $\lambda_{0}=811 \mathrm{~nm}$ for Au was set to ${ }^{48} n_{\mathrm{Au}}=0.18378-$ $i 5.2301$, and the buffer solution cladding was set to $n_{\text {clad }}=1.32934-$ $i 1.5008 \times 10^{-7}$, modified slightly from water to account for the buffer's salt content. ${ }^{49}$ The laser beam in the $3 \mathrm{D}$ models was an analyticdefined Gaussian beam that impinged on the V-groove mirror with the focal plane set to $y=0$ (groove vertex) and center at $x=-300 \mathrm{~nm}$ (partway back along the mirror and corresponding to maximum CPP in-coupling). Steady-state temperature distributions were solved for by coupling the electromagnetic wave (emw) model to the heat transfer (ht) model. Fixed temperature outer boundaries of $293.15 \mathrm{~K}$ were imposed at least $25 \mu \mathrm{m}$ distant from the spot center and $50 \mu \mathrm{m}$ distant along the V-groove axis [model size: $77.5 \mu \mathrm{m} \times 54 \mu \mathrm{m} \times 51.7 \mu \mathrm{m}$ $(x, y, z)]$ to ensure result convergence. The mesh element densities in the materials were set to maintain at least five elements per wavelength in the emw calculation, with an increasing density up to 20 elements per wavelength approaching the bottom of the $\mathrm{V}$-groove in the $\mathrm{Au}$ and liquid-cladding media. The model accounts for heat generation by the material absorption of light by including the Palik ${ }^{48}$ imaginary components of the refractive indices and using standard thermal properties such as heat capacities, thermal conductivities, etc., available from the COMSOL material library. The $5 \mathrm{~nm}$ chromium layer was approximated to the properties of gold to reduce meshing challenges and calculation memory. The light-to-heat efficiencies normalized to the laser input power were obtained by first integrating the normal heat flux magnitude exiting all outer boundaries of the V-groove model to find the total efficiency, $H_{\text {tot. }}$. This procedure was repeated with a separate, similar model but with the laser focusing on a flat gold surface to remove the presence of plasmonic excitation in order to find the normalized light-heat efficiency contribution from material absorption, $H_{\text {mat }}$. The heating efficiency caused by plasmonic loss was then obtained from $H_{\text {tot }}=H_{\text {plas }}+H_{\text {mat }}$.

\section{ASSOCIATED CONTENT}

\section{S Supporting Information}

The Supporting Information is available free of charge on the ACS Publications website at DOI: 10.1021/acsnano.6b08563.

Video 1: device operating principle (AVI)

Video 2: loading of DNA in the V-groove entropic traps (AVI)

Data and derivation of the experimentally measured drift and friction coefficient of $\lambda$-DNA in the nanoslitembedded V-grooves; additional numerical simulations presenting the cross-sectional heat flux and influence of the gold layer thickness on heat generation; fabrication process schematic and optical images of resulting devices; experimental setup schematic for temperature mapping, data of the thermal profile with the beam on a flat gold section, normalized spectra of filtering components for DNA imaging plus photothermal transport; discussion of DNA propulsion forces (PDF)

\section{AUTHOR INFORMATION}

\section{Corresponding Author}

*E-mail: anders.kristensen@nanotech.dtu.dk.

ORCID

Cameron L. C. Smith: 0000-0002-4048-827X

Jonas N. Pedersen: 0000-0001-7642-8212 


\section{Author Contributions}

C.L.C.S. and A.H.T. conceived the experiments. C.L.C.S., A.H.T., and R.M. performed the plasmonic and photothermal experiments. C.L.C.S., T.H.Y., J.C.D., N.A.M., and R.M. performed the DNA trapping and diffusion experiments. C.L.C.S., J.N.P., A.H.T., R.M., and A.K. analyzed the results. C.L.C.S. implemented the numerical simulations. C.L.C.S. and A.H.T. developed and carried out the device fabrication process. C.L.C.S., A.H.T., and J.N.P. wrote the first draft of the manuscript and prepared the figures. All authors discussed the results and contributed to the final version.

\section{Notes}

The authors declare no competing financial interest.

\section{ACKNOWLEDGMENTS}

C.L.C.S. acknowledges support from the Danish Council for Independent Research (Grant No. 12-126601). J.N.P. and R.M. acknowledge support from the EU project Cell-O-Matic (Grant No. 278204). R.M. acknowledges support from the Danish Council for Strategic Research (Grant No. 10-092322). A.K. acknowledges partial financial support from the Innovation Fund Denmark (Grant. No 10-092322) and from the European Union's Horizon 2020 research and innovation programme (Grant No. 665233). We thank Johan Eriksen for assistance with the temperature mapping measurements.

\section{REFERENCES}

(1) Reisner, W.; Morton, K.; Riehn, R.; Wang, Y.; Yu, Z.; Rosen, M.; Sturm, J.; Chou, S.; Frey, E.; Austin, R. Statics and Dynamics of Single DNA Molecules Confined in Nanochannels. Phys. Rev. Lett. 2005, 94, 196101.

(2) Turner, S.; Cabodi, M.; Craighead, H. Confinement-Induced Entropic Recoil of Single DNA Molecules in a Nanofluidic Structure. Phys. Rev. Lett. 2002, 88, 128103.

(3) Craighead, H. Future Lab-on-a-Chip Technologies for Interrogating Individual Molecules. Nature 2006, 442, 387-393.

(4) Reisner, W.; Pedersen, J. N.; Austin, R. H. DNA Confinement in Nanochannels: Physics and Biological Applications. Rep. Prog. Phys. 2012, 75, 106601.

(5) Reisner, W.; Larsen, N. B.; Silahtaroglu, A.; Kristensen, A.; Tommerup, N.; Tegenfeldt, J. O.; Flyvbjerg, H. Single-Molecule Denaturation Mapping of DNA in Nanofluidic Channels. Proc. Natl. Acad. Sci. U. S. A. 2010, 107, 13294.

(6) Marie, R.; Pedersen, J. N.; Bauer, D. L. V; Rasmussen, K. H.; Yusuf, M.; Volpi, E.; Flyvbjerg, H.; Kristensen, A.; Mir, K. U. Integrated View of Genome Structure and Sequence of a Single DNA Molecule in a Nanofluidic Device. Proc. Natl. Acad. Sci. U. S. A. 2013, 110, 4893-4898.

(7) Han, J.; Turner, S.; Craighead, H. Entropic Trapping and Escape of Long DNA Molecules at Submicron Size Constriction. Phys. Rev. Lett. 1999, 83, 1688-1691.

(8) Han, J.; Craighead, H. G. Separation of Long DNA Molecules in a Microfabricated Entropic Trap Array. Science 2000, 288, 1026-1029.

(9) Reisner, W.; Larsen, N. B.; Flyvbjerg, H.; Tegenfeldt, J. O.; Kristensen, A. Directed Self-Organization of Single DNA Molecules in a Nanoslit via Embedded Nanopit Arrays. Proc. Natl. Acad. Sci. U. S. A. 2009, 106, 79-84.

(10) Mikkelsen, M. B.; Reisner, W.; Flyvbjerg, H.; Kristensen, A. Pressure-Driven DNA in Nanogroove Arrays: Complex Dynamics. Nano Lett. 2011, 11, 1598-1602.

(11) Marie, R.; Kristensen, A. Nanofluidic Devices towards Single DNA Molecule Sequence Mapping. J. Biophotonics 2012, 5, 673-686.

(12) Yeh, J.-W.; Taloni, A.; Chen, Y.-L.; Chou, C.-F. Entropy-Driven Single Molecule Tug-of-War of DNA at Micro-Nanofluidic Interfaces. Nano Lett. 2012, 12, 1597-1602.
(13) Roxworthy, B. J.; Ko, K. D.; Kumar, A.; Fung, K. H.; Chow, E. K. C.; Liu, G. L.; Fang, N. X.; Toussaint, K. C. Application of Plasmonic Bowtie Nanoantenna Arrays for Optical Trapping, Stacking, and Sorting. Nano Lett. 2012, 12, 796-801.

(14) Zheng, Y.; Ryan, J.; Hansen, P.; Cheng, Y.-T.; Lu, T.-J.; Hesselink, L. Nano-Optical Conveyor Belt, Part II: Demonstration of Handoff Between Near-Field Optical Traps. Nano Lett. 2014, 14, 2971-2976.

(15) Juan, M. L.; Righini, M.; Quidant, R. Plasmon Nano-Optical Tweezers. Nat. Photonics 2011, 5, 349-356.

(16) Maragò, O. M.; Jones, P. H.; Gucciardi, P. G.; Volpe, G.; Ferrari, A. C. Optical Trapping and Manipulation of Nanostructures. Nat. Nanotechnol. 2013, 8, 807-819.

(17) Erickson, D.; Serey, X.; Chen, Y.-F.; Mandal, S. Nanomanipulation Using near Field Photonics. Lab Chip 2011, 11, 9951009.

(18) Donner, J. S.; Baffou, G.; McCloskey, D.; Quidant, R. PlasmonAssisted Optofluidics. ACS Nano 2011, 5, 5457-5462.

(19) Ndukaife, J. C.; Kildishev, A. V.; Nnanna, A. G. A.; Shalaev, V. M.; Wereley, S. T.; Boltasseva, A. Long-Range and Rapid Transport of Individual Nano-Objects by a Hybrid Electrothermoplasmonic Nanotweezer. Nat. Nanotechnol. 2015, 11, 53-59.

(20) Ndukaife, J. C.; Mishra, A.; Guler, U.; Nnanna, A. G. A.; Wereley, S. T.; Boltasseva, A. Photothermal Heating Enabled by Plasmonic Nanostructures for Electrokinetic Manipulation and Sorting of Particles. ACS Nano 2014, 8, 9035-9043.

(21) Cuche, A.; Canaguier-Durand, A.; Devaux, E.; Hutchison, J. A.; Genet, C.; Ebbesen, T. W. Sorting Nanoparticles with Intertwined Plasmonic and Thermo-Hydrodynamical Forces. Nano Lett. 2013, 13, $4230-4235$.

(22) Garcés-Chávez, V.; Quidant, R.; Reece, P. J.; Badenes, G.; Torner, L.; Dholakia, K. Extended Organization of Colloidal Microparticles by Surface Plasmon Polariton Excitation. Phys. Rev. B: Condens. Matter Mater. Phys. 2006, 73, 085417.

(23) Baffou, G.; Quidant, R. Thermo-Plasmonics: Using Metallic Nanostructures as Nano-Sources of Heat. Laser Photon. Rev. 2013, 7, $171-187$.

(24) Bozhevolnyi, S.; Volkov, V.; Devaux, E.; Ebbesen, T. Channel Plasmon-Polariton Guiding by Subwavelength Metal Grooves. Phys. Rev. Lett. 2005, 95, 046802.

(25) Smith, C. L. C.; Stenger, N.; Kristensen, A.; Mortensen, N. A.; Bozhevolnyi, S. I. Gap and Channeled Plasmons in Tapered Grooves: A Review. Nanoscale 2015, 7, 9355-9386.

(26) Bozhevolnyi, S. I.; Volkov, V. S.; Devaux, E.; Laluet, J.-Y.; Ebbesen, T. W. Channel Plasmon Subwavelength Waveguide Components Including Interferometers and Ring Resonators. Nature 2006, 440, 508-511.

(27) Volkov, V. S.; Bozhevolnyi, S. I.; Devaux, E.; Laluet, J.-Y.; Ebbesen, T. W. Wavelength Selective Nanophotonic Components Utilizing Channel Plasmon Polaritons. Nano Lett. 2007, 7, 880-884.

(28) Burgos, S. P.; Lee, H. W.; Feigenbaum, E.; Briggs, R. M.; Atwater, H. A. Synthesis and Characterization of Plasmonic Resonant Guided Wave Networks. Nano Lett. 2014, 14, 3284-3292.

(29) Vernon, K. C.; Tischler, N.; Kurth, M. L. Coupling of Energy from Quantum Emitters to the Plasmonic Mode of V Groove Waveguides: A Numerical Study. J. Appl. Phys. 2012, 111, 064323.

(30) Bermúdez-Ureña, E.; Gonzalez-Ballestero, C.; Geiselmann, M.; Marty, R.; Radko, I. P.; Holmgaard, T.; Alaverdyan, Y.; Moreno, E.; Garcia-Vidal, F. J.; Bozhevolnyi, S. I.; Quidant, R. Coupling of Individual Quantum Emitters to Channel Plasmons. Nat. Commun. 2015, 6, 7883.

(31) Li, Q.; Zhang, W.; Zhao, H.; Qiu, M. Two-Dimensional Analysis Photothermal Properties in Nanoscale Plasmonic Waveguides for Optical Interconnect. J. Lightwave Technol. 2013, 31, 4051-4056.

(32) Duhr, S.; Braun, D. Why Molecules Move along a Temperature Gradient. Proc. Natl. Acad. Sci. U. S. A. 2006, 103, 19678-19682.

(33) Thamdrup, L. H.; Larsen, N. B.; Kristensen, A. Light-Induced Local Heating for Thermophoretic Manipulation of DNA in Polymer Micro- and Nanochannels. Nano Lett. 2010, 10, 826-832. 
(34) He, Y.; Tsutsui, M.; Scheicher, R. H.; Bai, F.; Taniguchi, M.; Kawai, T. Thermophoretic Manipulation of DNA Translocation through Nanopores. ACS Nano 2013, 7, 538-546.

(35) Belkin, M.; Maffeo, C.; Wells, D. B.; Aksimentiev, A. Stretching and Controlled Motion of Single-Stranded DNA in Locally Heated Solid-State Nanopores. ACS Nano 2013, 7, 6816-6824.

(36) Pedersen, J. N.; Lüscher, C. J.; Marie, R.; Thamdrup, L. H.; Kristensen, A.; Flyvbjerg, H. Thermophoretic Forces on DNA Measured with a Single-Molecule Spring Balance. Phys. Rev. Lett. 2014, 113, 268301.

(37) Li, Q.; Liu, G.; Yang, H.; Wang, W.; Luo, S.; Dai, S.; Qiu, M. Optically Controlled Local Nanosoldering of Metal Nanowires. Appl. Phys. Lett. 2016, 108, 193101.

(38) Roxworthy, B. J.; Bhuiya, A. M.; Vanka, S. P.; Toussaint, K. C. Understanding and Controlling Plasmon-Induced Convection. Nat. Commun. 2014, 5, 3173.

(39) Tegenfeldt, J. O.; Prinz, C.; Cao, H.; Chou, S.; Reisner, W. W.; Riehn, R.; Wang, Y. M.; Cox, E. C.; Sturm, J. C.; Silberzan, P.; Austin, R. H. The Dynamics of Genomic-Length DNA Molecules in 100-nm Channels. Proc. Natl. Acad. Sci. U. S. A. 2004, 101, 10979-10983.

(40) Quake, S. R.; Babcock, H.; Chu, S. The Dynamics of Partially Extended Single Molecules of DNA. Nature 1997, 388, 151-154.

(41) Smith, C. L. C.; Thilsted, A. H.; Garcia-Ortiz, C. E.; Radko, I. P.; Marie, R.; Jeppesen, C.; Vannahme, C.; Bozhevolnyi, S. I.; Kristensen, A. Efficient Excitation of Channel Plasmons in Tailored, UVLithography-Defined V-Grooves. Nano Lett. 2014, 14, 1659-1664.

(42) Søndergaard, T.; Novikov, S. M.; Holmgaard, T.; Eriksen, R. L.; Beermann, J.; Han, Z.; Pedersen, K.; Bozhevolnyi, S. I. Plasmonic Black Gold by Adiabatic Nanofocusing and Absorption of Light in Ultra-Sharp Convex Grooves. Nat. Commun. 2012, 3, 969.

(43) Jorge, P. A. S.; Maule, C.; Silva, A. J.; Benrashid, R.; Santos, J. L.; Farahi, F. Dual Sensing of Oxygen and Temperature Using Quantum Dots and a Ruthenium Complex. Anal. Chim. Acta 2008, 606, 223229.

(44) Bjorndal, M. T.; Fygenson, D. K. DNA Melting in the Presence of Fluorescent Intercalating Oxazole Yellow Dyes Measured with a Gel-Based Assay. Biopolymers 2002, 65, 40-44.

(45) Siegert, S.; Friedrich, R; Peinke, J. Analysis of Data Sets of Stochastic Systems. Phys. Lett. A 1998, 243, 275-280.

(46) Friedrich, R.; Siegert, S.; Peinke, J.; Lück, S.; Siefert, M.; Lindemann, M.; Raethjen, J.; Deuschl, G.; Pfister, G. Extracting Model Equations from Experimental Data. Phys. Lett. A 2000, 271, 217-222.

(47) Bilenberg, B.; Nielsen, T.; Clausen, B.; Kristensen, A. PMMA to SU-8 Bonding for Polymer Based Lab-on-a-Chip Systems with Integrated Optics. J. Micromech. Microeng. 2004, 14, 814-818.

(48) Palik, E. D. Handbook of Optical Constants of Solids; Academic Press: San Diego, CA, 1985.

(49) Dorsey, N. E. Properties of Ordinary Water-Substance; American Chemical Society: Washington, DC, 1940; Vol. 81, p 673. 